\title{
A singular value sensitivity approach to robust eigenstructure assignment
}

\author{
Søgaard-Andersen, Per; Trostmann, Erik; Conrad, Finn
}

Published in:

25th IEEE Conference on Decision and Control

Link to article, DOI:

10.1109/CDC.1986.267168

Publication date:

1986

Document Version

Publisher's PDF, also known as Version of record

Link back to DTU Orbit

Citation $(A P A)$ :

Søgaard-Andersen, P., Trostmann, E., \& Conrad, F. (1986). A singular value sensitivity approach to robust eigenstructure assignment. In 25th IEEE Conference on Decision and Control (pp. 121-126). IEEE. https://doi.org/10.1109/CDC.1986.267168

\section{General rights}

Copyright and moral rights for the publications made accessible in the public portal are retained by the authors and/or other copyright owners and it is a condition of accessing publications that users recognise and abide by the legal requirements associated with these rights.

- Users may download and print one copy of any publication from the public portal for the purpose of private study or research.

- You may not further distribute the material or use it for any profit-making activity or commercial gain

- You may freely distribute the URL identifying the publication in the public portal 


\title{
A SINGULAR VALUE SENSITIVITY APPROACH TO ROBUST EIGENSTRUCTURE ASSIGNMENT
}

\author{
Per Søgaard-Andersen \\ Erik Trostmann \\ Finn Conrad
}

Control Engineering Institute

Technical University of Denmark

Building 424, 2800 Lyngby - Denmark

\section{Abstract}

A design technique for improving the feedback properties of multivariable state feedback systems designed using eigenstructure assignment is presented. Based on a singular value analysis of the feedback properties a design parameter adjustment procedure is outlined. This procedure allows for the preservation of important properties of the initial design, by selecting only a subset of the design parameters for adjustment.

The computational requirements are modest and straight-forward to implement with standard software. An example illustrating the method is included.

\section{Introduction}

Recent deveiopments have showed that eigenstructure assignment in multivariable systems is well-suited for obtaining specified I/O-response properties [1-5]. Unfortunately this ability is not followed by any significant robustness guarantees comparable to LQ-guarantees. Here the robustness measure is the minimal singular value of the return difference or inverse return difference matrix [6-8]. In practice it may therefore be necessary tc "recover" acceptable robustness properties like the multivariable stability margins. In this "recovery" procedure it is important that significant properties of the initial design are preserved.

In this paper an approach to this recovery problem is presented. The recovery information used is the singular value sensitivities of the robustness measures taken at the frequencies where robustness should be improved. These sensitivities indicate the directions the design parameters should be changed towards, to ensure the robustness improvement. By selecting only a subset of the design parameters for adjustment it is possible to preserve significant aspects of the initial design.

The adjustment of the design parameters can be performed by forma: optimization methods or by direct tuning.

Earlier approaches to robustness improvement using eigenstructure assignment have focused on a time-domain characterization of the robustness problem, and have aimed towards improving the spectral condition number of the closed-10op eigenstructure [16, and references herein]. The approach in this paper differs from these approaches since the robustness problem is viewed from a frequency-domain perspective.

Other applications of singular value sensitivities for robustness improvement based on other design methodologies nay be found in refs. $[10,12]$.

The outline of the paper is as follows. In section two - eigenstructure assignment and the singular value based robustness concepts are shortly reviewed, in section three the singular value sensitivities are derived. In section four the adjustment procedure is outlined and the computational aspects discussed. The method is illustrated by an example in section five and a few summarizing remarks follow in section 6.

\section{The problem statement}

Consider the finite-dimensional linear time-invariant system

$$
\dot{x}=\mathrm{Ax}+\mathrm{Bu}, \quad \mathrm{x} \in \mathbb{R}^{\mathrm{n}}, u \in \mathbb{R}^{\mathrm{m}}
$$

where $A, B$ are constant matrices of appropriate dimensions. $B$ is assumed to be of full rank. ( $A, B$ ) is a controllable pair.

It is desired to design a full state feedback control law

$$
u=-K x+r, \quad r \in \mathbb{R}^{m}
$$

In the context of eigenstructure assignment the extra freedom inherent in multivariable state feedback (beyond eigenvalue placement) is utilized to place parts of the closed-loop eigenvectors.

\section{2.i Eigenstructure assignment}

The extra freedom of selecting eigenvectors may be summarized as [1]:

Let $\Lambda=\left\{\lambda_{i}\right\}_{i=1}^{\pi}$ be a self-conjugate set of distinct complex numbers containing no open-loop eigenvalues. There exists a real matrix $K$ such that $v_{i}$ is an eigenvector of $A-B K$ iff.

a) $\left\{v_{i}\right\}_{i=1}^{n}$ are linearly independent in $c^{n}$ and

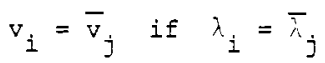

b) $\left[\begin{array}{l}v_{i} \\ t_{i}\end{array}\right] \in \operatorname{Ker}\left[\lambda_{i} I-A,-B\right]$

c) $\mathrm{K}=-\left[\mathrm{t}_{1} \ldots \mathrm{t}_{\mathrm{n}}\right] \cdot\left[\mathrm{v}_{1} \ldots \mathrm{v}_{\mathrm{n}}\right]^{-1}=-\mathrm{TV}^{-1}$

The design parameters of this approach are the vectors $t_{i}$ and the set $\Lambda . V$ denotes the closed-loop eigenvector-matrix.

Several approaches to utilize this result in $\mathrm{I} / \mathrm{O}$ response design have been derived [1-5].

unfortunately the resulting designs have no significant guaranteed robustness properties, when the robustress measure is the minimum singular value of a return difference or inverse return difference matrix [6-8]. If the resulting robustness properties evaluated over an appropriate frequency - interval is unsatisfactory, some method for "recovering" acceptable robustness levels is necessary. If possible this method should preserve as many of the desirable I/O-response properties of the initial design as possible. 
In section 3 a technique dealing with this problem is developed. In the next section a short review of the singular value-based robustness approach is given.

\subsection{Singular value-based robustness concepts}

The robustness of a control loop with respect to a given loop breaking point can be described by the minimum singular value of the associated return difference or inverse return difference matrix evaluated over the frequencies [6-8].

Stability robustness is tested by the condition $[6-7]$.

$$
\bar{\sigma}(L(j \omega))<\underline{\sigma}\left(I+G^{-1}(j \omega)\right) \quad \forall \omega \geq 0
$$

where $L(j \omega)$ represents the multiplicative uncertainties at the loop-breaking point, and $G(j \omega)$ is the loop transfer. $\sigma$ is the minimum singular value of the argument.

The stability margins are defined by [7-8].

$$
\inf \sigma(I+G(j \omega)) \text { and } \inf _{\omega} \underline{\sigma}\left(I+G^{-1}(j \omega)\right)
$$

A second robustness objective concerns the loop performance. The formulation of this issue in the singular value setting is discussed in $[6,11]$.

A detailed account of these results is given in the original references.

\section{Singular value sensitivities in eigenstructure assignment}

Let the singular value decomposition (SVD) of a complex matrix $M$ be

$$
\begin{aligned}
& M(p)=U(p) \sum(p) Z(p)^{H}, \quad \sum=\operatorname{diag}\left(\sigma_{i}\right) \\
& \mathrm{UU}^{\mathrm{H}}=I, \quad \mathrm{zz}^{\mathrm{H}}=\mathrm{I}, \quad \sigma_{1}>\ldots>\sigma_{\mathrm{m}} \geqslant 0
\end{aligned}
$$

Here $\sigma_{i}$ denotes the singular values, $U$ and $z$ contain the associated singular vectors. A detailed outline of the SVD is given in [18].

The variable $p$ in $(3-1)$ denotes a real parameter. In the following $M$ is assumed to be analytic in $p$. The sensitivity (or gradient) of $\sigma_{i}[M(p)]$ with respect to $p$ is for distinct $\sigma_{i}$

$$
\frac{\partial \sigma_{i}}{\partial p}=\operatorname{Re}\left[u_{i}{ }^{\mathrm{H}} \frac{\partial M}{\partial p} z_{i}\right]
$$

with Re as the real part of the argument.

Let $\Omega=\left\{\omega_{e}\right\}_{e=1}^{q}$ denote a set of frequencies where an initial design has unsatisfactory robustness properties. One systematic approach to the redesign of the controller is to evaluate the singular value sensitivities with respect to the design parameters at the frequencies $\Omega$. The sensitivities will indicate in which directions the design parameters should be moved to improve the robustness characteristics.

The design parameters in eigenstructure assignment are the parameter vectors $t_{i}$ and the eigenvalues $\lambda_{i}$.

In this section singular value sensitivities associated with these design parameters are derived.

only return difference matrices for the loop broken at the input are considered. Results for other robustness measures are given in appendix $A$. Furthermore it is assumed that the design parameters $t_{i}$ and $\lambda_{i}$ are real, the complex case is treated in appendix $B$.

\subsection{Design parameters $t_{i}$}

Let $p$ be the $j^{\prime}$ th element of $t_{i}$, and let $M$ be the return difference taken at the frequency $\omega=\omega_{e}$.

$$
\begin{aligned}
& M \quad=I+G=I+X \psi\left(j \omega_{e}\right) \\
& \psi\left(j \omega_{e}\right)=\left(\left(j \omega_{e}\right) I-A\right)^{-1} B=\Phi\left(j \omega_{e}\right) B
\end{aligned}
$$

In the following the explicit dependency of $p$ and $\omega_{e}$ is suppressed.

The sensitivity of $\underline{\sigma}(I+G)$ at $\omega=\omega_{e}$ with respect to $t_{i j}$ is

$$
\begin{aligned}
& \frac{\partial \sigma}{\partial t_{i j}}=\operatorname{Re}\left[\underline{\underline{u}} \frac{\partial(I+G)}{\partial t_{i j}} \underline{z}\right] \\
& \frac{\partial(I+G)}{\partial t_{i j}}=\frac{\partial K}{\partial t_{i j}} \psi
\end{aligned}
$$

Since $\mathrm{KV}=-\mathrm{T}$ the following applies

$$
\begin{aligned}
\frac{\partial K}{\partial t_{i j}} & =\left[-\frac{\partial T}{\partial t_{i j}}-k \frac{\partial V}{\partial t_{i j}}\right] v^{-1} \\
\frac{\partial T}{\partial t_{i j}} & =\left[\begin{array}{llllll}
{\left[0 \ldots 0 e_{j}\right.} & \ldots & 0
\end{array}\right]
\end{aligned}
$$

$$
\begin{aligned}
& \frac{\partial v}{\partial t_{i j}}=\left[\begin{array}{lllll}
0 & \ldots & \frac{\partial v_{i}}{\partial t_{i j}} & \ldots & \ldots
\end{array}\right]
\end{aligned}
$$

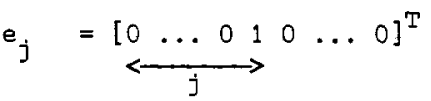

from eq. $(2-3)$ it is found that $v_{i}=\Phi\left(\lambda_{i}\right) B t_{i}$ and thus

$$
\begin{aligned}
& \frac{\partial v_{i}}{\partial t_{i j}}=\Phi\left(\lambda_{i}\right) b_{j} \\
& \frac{\partial k}{\partial t_{i j}}=-\left(e_{j}+K \Phi\left(\lambda_{i}\right) b_{j}\right) w_{i}^{T}
\end{aligned}
$$

where $w_{i}{ }^{T}$ is the $i$ 'th left eigenvector of $A-B K$. If eq. $(3-6)$ is inserted in eq. (3-4) the singular value sensitivity is given by

$$
\frac{\partial \sigma}{\partial t_{i j}}=\operatorname{Re}\left[\underline{u}^{\mathrm{H}}\left(e_{j}+K \Phi\left(\lambda_{i}\right) b_{j}\right) w_{i}^{T} \psi\left(j \omega_{e}\right) \underline{z}\right]
$$

and the gradient of $\sigma$ with respect to $T$ is

$$
\begin{aligned}
& \nabla_{T^{K}}=\left[\begin{array}{ccc}
\frac{\partial K}{\partial t_{11}} & \cdots & \frac{\partial K}{\partial t_{1 n}} \\
\vdots & & \vdots \\
\frac{\partial K}{\partial t_{m 1}} & \cdots & \frac{\partial K}{\partial t_{m n}}
\end{array}\right] \\
& \nabla_{\mathrm{T}}-\left.\right|_{\omega_{\mathrm{e}}}=\operatorname{Re}\left[\mathrm{U}_{\mathrm{B}} \cdot \nabla_{\mathrm{T}} \mathrm{K} \cdot \cdot \mathrm{z}_{\psi \mathrm{B}}\right] \\
& \mathrm{U}_{\mathrm{B}}=\text { Blockdiag }\left(\underline{u}^{\mathrm{H}}\right) \\
& z_{\psi B}=\text { Blockdiag }\left(\psi\left(j \omega_{e}\right) \underline{z}\right)
\end{aligned}
$$

with $\operatorname{dim} U_{B}=m x \mathbb{m}^{2}$ and $\operatorname{dim} z_{\psi_{B}}=n^{2} \times n$. 
3.2 Design parameters $\lambda_{i}$

If $p$ is selected as a distinct closed-loop eigenvalue $\lambda_{i}$ the following results apply.

$$
\begin{aligned}
& \frac{\partial(I+G)}{\partial \lambda_{i}}:_{\omega_{e}}=\frac{\partial K}{\partial \lambda_{i}} \psi\left(j \omega_{e}\right) \\
& \frac{\partial K}{\partial \lambda_{i}}=-k \frac{\partial v}{\partial \lambda_{i}} v^{-1}
\end{aligned}
$$

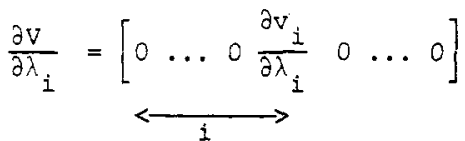

Since $v_{i}=\oint\left(\lambda_{i}\right) B t_{i}$ then

$$
\begin{aligned}
& \frac{\partial v_{i}}{\partial \lambda_{i}}=\frac{\partial \Phi\left(\lambda_{i}\right)}{\partial \lambda_{i}} B t_{i} \\
& \frac{\partial \Phi\left(\lambda_{i}\right)}{\partial \lambda_{i}}=-\Phi\left(\lambda_{i}\right) \frac{\partial \Phi^{-1}\left(\lambda_{i}\right)}{\partial \lambda_{i}} \Phi\left(\lambda_{i}\right)=-\Phi\left(\lambda_{i}\right)^{2}
\end{aligned}
$$

$$
\frac{\partial v_{i}}{\partial \lambda_{i}}=-\Phi\left(\lambda_{i}\right) v_{i}
$$

and thus

$$
\frac{\partial \mathrm{K}}{\partial \lambda_{i}}=K \Phi\left(\lambda_{i}\right) v_{i} w_{i}^{T}
$$

where $w_{i}^{T}$ is the $i$ 'th left eigenvector of $A-B K$ and

$$
\left.\frac{\partial \sigma}{\partial \lambda_{i}}\right|_{\omega_{e}}=\operatorname{Re}\left[\underline{\underline{u}}^{\mathrm{H}} \mathrm{K} \Phi\left(\lambda_{i}\right) v_{i} \omega_{i} \mathrm{~T} \psi\left(j \omega_{e}\right) \underline{z}\right]
$$

The gradient of $\underline{\sigma}(I+G)$ with respect to $\Lambda$ is

$$
\begin{aligned}
& \nabla_{\Lambda} \mathrm{K}=\left[\frac{\partial \mathrm{K}}{\partial \lambda_{1}}, \cdots, \frac{\partial \mathrm{K}}{\partial \lambda_{\mathrm{n}}}\right] \\
& \nabla_{\Lambda-\sigma} \omega_{e}=\operatorname{Re}\left[\underline{u}^{\mathrm{H}} \cdot \nabla_{\Lambda} \mathrm{K} \cdot \mathrm{z}_{\psi \mathrm{B}}\right]
\end{aligned}
$$

The results of sections 3.1 and 3.2 concerning singular value gradients may be summarized as:

\section{Proposition}

Let $\sigma$ be a distinct minimal singular value of $\mathrm{M}=$ $I+K\left(j \omega_{e} I-A\right)^{-1} B$, where $K=K(T, \Lambda)$ and $M$ is analytic in the elements of $T$ and $\Lambda$. (A, B) is a controllable pair, and let $u$ and $z$ denote the left and right singular vectors associated with $\sigma . \Lambda$ is a set of distinct complex conjugate numbers which does not contain any members of the spectrum of $A$. Then the gradients of $\sigma$ with respect to the design parameters $T$ and $\Lambda$ evaluated at $s=j \omega_{e}$ are

$$
\begin{aligned}
& \nabla_{\mathrm{T}} \sigma(I+\mathrm{K} \Phi \mathrm{B})=\operatorname{Re}\left[\underline{\mathrm{U}}_{\mathrm{B}} \cdot \nabla_{\mathrm{T}} \mathrm{K} \cdot \mathrm{z}_{\psi \mathrm{B}}\right] \\
& \nabla_{\Lambda} \underline{\sigma}(I+\mathrm{K} \Phi \mathrm{B})=\operatorname{Re}\left[\underline{\underline{U}}^{\mathrm{H}} \cdot \nabla_{\Lambda} \mathrm{K} \cdot \mathrm{Z}_{\psi \mathrm{B}}\right]
\end{aligned}
$$

Remark 1. If $\sigma$ is not a distinct singular value the concept of generalized gradients can be applied. This requires the Gateaux differentials, as discussed in [9].
Remark 2. When the elements $t_{i j}$ and $\lambda_{i}$ are complex the corresponding expressions $\nabla_{T} k$ and $\nabla_{\Lambda} k$ are given in appendix $B$.

Remark 3. Gradients according to other loop-breaking points and other robustness measures are given in appendix A.

Remark 4. The inclusion of multiple eigenvalues in $A$

does not pose any principal problem, but it is computationally tedious. Anyway this is not justified from robustness considerations [16].

Remark 5. Open-loop eigenvalues of $A$ and uncontrollable eigenvalues can be included in the set $A$. This requires slightly modified methods, as discussed in [17].

In section 4 the design adjustment procedure is outined.

\subsection{Design parameter adjustment procedure}

The gradients $\nabla_{T} \sigma$ and $\nabla_{\Lambda} \sigma$ indicates the directions the design parameters should be adjusted towards to improve the robustness characteristics. In many situations it is not acceptable to adjust all design parameters since some of these correspond to desirable characteristics. In eigenstructure assignment some vectors $t_{i}$ and the associated eigenvalues $\lambda_{i}$ might have

been chosen to obtain certain dominant response characteristics and certain "mode-mixing" i.e. a desirable I/O-response, whereas other vectors $t_{i}$ and $\lambda_{i}$ are less important/dominant from the I/O-point of view. These design parameters should then be chosen for adjustment in order to obtain good robustness properties.

If the measure $\sigma(I+G)$ evaluated of some frequency $\omega_{e}$ is too small, then the design parameters selected for adjustment (adjustment parameters) should be reselected as

$$
\begin{array}{ll}
t_{i j}=t_{i j}^{0}+\varepsilon_{i j} \frac{\partial \sigma}{\partial t_{i j}} & \varepsilon_{i j} \geqq 0 \\
\lambda_{i}=\lambda_{i}^{0}+\varepsilon_{i} \frac{\partial \sigma}{\partial t_{i j}} & \varepsilon_{i} \geqq 0
\end{array}
$$

where $\varepsilon_{i j}$ and $\varepsilon_{i}$ are adjustment parameters which are selected by the designer. Since only first order sensitivities are considered the parameters $\varepsilon_{i j}$ and $\varepsilon_{i}$ should be chosen carefully to guarantee an improvement of $\sigma(I+G)$.

If $\sigma(I+G)$ and possibly also other singular value quantities should be adjusted at several frequencies, and furthermore some quantities must not decrease at other frequencies, the problem of finding feasible solutions $\Delta t_{i j}$ and $\Delta \lambda_{i}$ is less transparent. In this case the problem may be formulated as a linear programming problem as discussed in [10], or as a formal optimization problem.

one such formulation is based on the index [12]

$$
J=\sum_{i=1}^{N}\left[\max \left\{0, \underline{\sigma}_{D}\left(j \omega_{i}\right)-\underline{\sigma}\left(j \omega_{i}\right)\right\}\right]^{2}
$$

where $\mathrm{N}$ is the total number of frequencies selected for adjustment and $\underline{\sigma}_{D}\left(j \omega_{i}\right)$ is the desired level at $\omega=\omega_{i}$.

By using the gradients developed in $\S 3.1$ and 3.2 the optimization can be performed. 
In [12] a constant level $\underline{\sigma}_{D}$ is used to improve the stability margins. If $\sigma_{D}\left(j \omega_{i}\right)$ is instead frequency dependent the optimization can be applied to multivariable loop-shaping [11], where $\sigma_{D}\left(j w_{i}\right)$ indicates the desired loop-shape.

The design procedure described above may be summarized in the following design principle

Step 1. Do the initial design with some eigenspace technique.

Step 2. Evaluate the stability robustness and the performance. If these are not acceptable go to step 3 .

Step 3. Select the frequencies where robustness should be improved, or should not be allowed to change. Evaluate the gradients $\nabla_{\mathrm{r}} \underline{\sigma}$ and $\nabla_{\Lambda} \underline{\sigma}$ at the frequencies where improvements are desired and for the relevant robustness quantities.

Step 4. Select the design parameters which are allowed to be adjusted and select the new set of adjustment parameters, $T_{\text {new }}$ and $\Lambda_{\text {new }}$ either by direct adjustment or with some optinization scheme. Evaluate the new controller K. Go to step 2 .

The method does not provide any guarantees concerning achievable singular value plots. The final controller can be very dependent on the nominal controller.

Notice that the design objectives are only obtainable if they are in accordance with fundamental performance limitations $[6,7]$.

\subsection{Computational and numerical issues}

The computational requirements for evaluation of the singular value gradients are modest, since the formulas involved are straight-forward to evaluate. All necessary computations may be performed using standard numerically well-proven software like MATLAB [14]. MATLAB also provides a relevant media for the interaction between the designer and the design software.

If the parameter adjustment is performed by some optimization scheme, standard software also exists for this task $[10,12]$.

If the singular values of a matrix are clustered the calculation of the singular vectors is ill-conditioned. Techniques for coping with this problem are considered in [9]. Efficient calculation of transfer function matrices is considered in [15].

The calculation of the vector space, which spans the subspace which $v_{i}$ belongs to, can be numerically illconditioned. For such cases the calculation of the assignable eigenvectors must be based on [4, 16]

$$
\left[\lambda_{i} I-A,-B\right]\left[\begin{array}{l}
v_{i} \\
t_{i}
\end{array}\right]=0
$$

The singular value decomposition of the first matrix in $(4-3)$ is

$$
\left[\lambda_{i} I-A,-B\right]=U \Sigma z^{H}
$$

$$
\begin{aligned}
& {\left[\lambda_{i} I-A,-B\right]\left[\begin{array}{ll}
z_{11 i} & z_{12 i} \\
z_{21 i} & z_{22 i}
\end{array}\right]=U\left[\begin{array}{llll}
\sigma_{1} & 0 & 1 & 0 \\
\ddots & \sigma_{n} & 1 & 0
\end{array}\right]} \\
& \underset{\mathrm{n}}{\longrightarrow} \underset{\mathrm{m}}{\longrightarrow} \underset{\mathrm{m}}{\longrightarrow}
\end{aligned}
$$

clearly

$$
\left[\begin{array}{l}
v_{i} \\
t_{i}
\end{array}\right]=\left[\begin{array}{l}
z_{12 i} \\
z_{221}
\end{array}\right] \alpha_{i}
$$

Based on this expression gradients can be evaluated. Details of this is outlined in [17].

\section{Example}

Consider the system (defined in [13]).

$$
\begin{aligned}
& A=\left[\begin{array}{lccc}
0 & 0.9945 & 0.1044 & 0 \\
0 & -1.525 & 0.0678 & -30.02 \\
0 & -0.0166 & -0.1502 & 5.159 \\
0.035 & 0.0698 & -0.9992 & -0.0903
\end{array}\right] \\
& B=\left[\begin{array}{cc}
0 & 0 \\
11.51 & 5.241 \\
0.1894 & -1.968 \\
-0.003 & 0.135
\end{array}\right]
\end{aligned}
$$

The initial design parameters have been selected as

$$
\begin{aligned}
& T_{0}=\left[\begin{array}{cccc}
4 & 1 & 1 & -1 \\
1 & 0 & 1 & 0
\end{array}\right] \\
& \Lambda_{0}=[-2.63 \pm j 3.26,-3.44 \pm j 1.60]
\end{aligned}
$$

In Figs. 1-3 the "nominal design" curve represents $\sigma(I+K \psi(j \omega))$. It is desired to improve the stability margins without deteriorating the nominal low frequency performance against disturbances. Therefore two frequencies $\omega_{1}=0.1 \mathrm{r} / \mathrm{s}$ and $\omega_{2}=10 \mathrm{r} / \mathrm{s}$ are selected as tuning frequencies, where $\nabla_{\mathrm{T}} \sigma$ and $\nabla_{\Lambda} \sigma$ are evaluated.

Three different sets of adjustment parameters are considered, and the associated robustness improvement is considered.

Case 1. All elements of $T$ are subject to adjustments, whereas $\Lambda$ should remain unchanged.

Case 2. All elements of $\Lambda$ are subject to adjustments, whereas $T$ should remain unchanged.

Case 3. $\lambda_{3}$ and $\lambda_{4}$ and $t_{3}$ and $t_{4}$ are subject to adjustments, whereas the other elements of $\Lambda$ and $T$ should remain unchanged.

The improvements in the three cases are shown in Figs. 1-3 in 4 steps. In all three cases the objectives are satisfied. The improvements in the multivariable stability margins (based on $\sigma(I+G)$ ) are summarized in Figure 4. Notice that in case 3 the stability robustness is almost equal to the LQ-margins, in the other two cases the margins are not much below LQ-margins. 
In case 2 the nominal performance is also increased significantly. In cases 1 and 3 the low frequency nomiral performance is almost equal to the initial value. In all 3 cases the price for the improvements is an increase in bandwidth and a change in $\mathrm{s}_{0}$ and $\Lambda_{0}$. The final values of $T$ and $\Lambda$ are in the 3 cases.

$$
\begin{aligned}
& \mathrm{T}_{1}=\left[\begin{array}{cccc}
4 & -0.6 & 1.2 & -1.6 \\
1.8 & 0.6 & 0.6 & 0
\end{array}\right] \quad \Lambda_{1}=\Lambda_{0} \\
& \mathrm{~T}_{2}=\mathrm{T}_{0} \Lambda_{2}=[-2.3 \pm j 3.0,-5 \pm j 4.5] \\
& \mathrm{T}_{3}=\left[\begin{array}{llll}
4 & 1 & 1 & -1.2 \\
1 & 0 & 0.5 & 0.4
\end{array}\right] \\
& \Lambda_{3}=[-2.63 \pm j 3.26,-4.8 \pm j 2.9] \\
& \underline{6 . \text { Summary }}
\end{aligned}
$$

In this paper a technique for improving insufficient robustness characteristics of control systems designed with eigenstructure assignment is considered. The technique is basec on singular value gradients evaluated at selected frequencies with respect to the design parameters. With this technique it is possible to select a subset of the design parameters for adjustments, while the remaining design parameters are not affected. This property implies that significant characteristics of the initial design remain unaffected, e.g. closedloop eigenvalues, mode-mixing and dominant behaviour. The formulas for evaluating the gradients are computationally simple and they are easily implemented with standard software.

The example illustrates that significant improvements can be obtained while important nominal design properties are maintained.

The methods in this paper are easily extended to the adjustment of observer design parameters, if these are formulated in the eigenstructure assignment framework.

The limitations of this technique are that gradient techniques do not necessarily find the "best" solutions to a given problem, unless it is used carefully. Furthermore, it is assumed that the robustness specifications can be expressed in the singular value-based context. More complex problems require the structured singular value [11].

\section{References}

[1] B.C. Moore: "On the flexibility offered by state feedback in multivariable systems beyond closedloop eigenvalue assignment", IEEE Transact. on Aut. Control 21, p. 689-92, 1976.

[2] A.N. Andry et. al: "Eigenstructure assignment in linear systems", IEEE Transact. Aerospace \& Electr. Syst., vol. 19, p. 711-29, 1983.

[3] T. Sadeghi et. al: "A control law for pole/ variant zero placement", American Control Conf. 1983, p. 912-7.

[4] T.B. Cunringham: "Eigenspace selection procedures for closed-loop response shaping with modal control", IEEE CDC 1980, p. 178-86.

[5] P. Søgaard-Andersen et. al: "Eigenstructure and residuals in multivariable state feedback design", Int. J. Control, vol. 44, p. 427-39, 1986.

[6] J.C. Doyle and G. Stein: "Multivariable feedback design: Concepts for a classical/modern synthesis", IEEE Transact. on Aut. Control 26, p. 4-26, 1981.

[7] M.G. Safonov et. al: "Feedback properties of multivariable systems: The role and use of the return difference matrix", IEEE Transact. on Aut. Control 26, p. 47-65, 1981.

[8] N.A. Lehtomaki et. al: "Robustness results in 1inear quadratic gaussion based multivariable control designs", IEEE Transact. on Aut. Control 26, p. $75-92,1981$.
[9] J.S. Freudenberg et. al: "Robustness analysis using singular value sensitivities", Int. J. Control, vol. 41, p. 1129-55, 1982.

[10] R.S. Mceven and D.P. Looze: "Quadratic weight adjustment for the enhancement of feedback properties", American Control Conf. 1982, p. 996-1001.

[11] G. Stein: "Beyond singular values and loop shapes". Rep. No. LIDS-P-1504, MIT Lab. of Information and Decision Systems, 1985.

[12] J.R. Newson and V. Mukhopadhyay: "A multiloop robust-controller design study using singular value gradients", J. Guidance vol. 8, p. 514-9, 1985.

[13] A.N. Andry et. al: "Robustness and eigenstructure in observer design", IEEE CDC 1983, p. 342-6.

[14] C. Moler: "MATLAB - An interactive matrix laboratory", University of New Mexico, 1980.

[15] A.J. Laub: "Efficient multivariable frequency response computations", IEEE Transact. on Aut. Control 26, p. 407-8, 1981.

[16] J. Kautsky: "Robust pole assignment in linear state feedback", Int. J. Control, vol. 41, p. 1129-55, 1985.

[17] P. Søgaard-Andersen, Ph.D. Thesis, Control Engineering Institute, DTE, Copenhagen, Denmark, 1986.

[18] P.C. Hansen: "SVD - Theory and Applications", Ph.D. Thesis, Numerical Institute, DTH, Copenhagen, Denmark, 1984 .

\section{Appendix A}

If the 10op-breaking point is at the plant output, the corresponding robustness measure is

$$
M=I+\psi\left(\omega_{e}\right) K=U \Sigma z^{H}
$$

and the corresponding gradients are

$$
\begin{aligned}
& \frac{\partial \underline{\sigma}}{\partial t_{i j}}=\operatorname{Re}\left[\underline{u}^{H} \psi\left(j \omega_{e}\right) \frac{\partial \mathrm{K}}{\partial t_{i j}} \underline{z}\right] \\
& \frac{\partial \underline{\sigma}}{\partial \lambda_{i}}=\operatorname{Re}\left[\underline{u}^{\mathrm{H}} \psi\left(j \omega_{e}\right) \frac{\partial \mathrm{K}}{\partial \bar{\lambda}_{i}} \underline{z}\right]
\end{aligned}
$$

If the inverse return difference matrix is used as robustness measure and the loops are broken at the input then

$$
\begin{aligned}
& M=I+\left(K \psi\left(j \omega_{e}\right)\right)^{-1}=U \Sigma z^{H} \\
& \frac{\partial \sigma}{\partial t_{i j}}=-\operatorname{Re}\left[U_{1}^{H} \frac{\partial K}{\partial t_{i j}} \psi\left(j \omega_{e}\right) z_{1}\right] \\
& \frac{\partial \sigma}{\partial \lambda_{i}}=-R e\left[U_{1}^{H} \frac{\partial K}{\partial \lambda_{i}} \psi\left(j \omega_{e}\right) z_{1}\right] \\
& u_{1}^{B}=\underline{u}^{H}\left(K \psi\left(j \omega_{e}\right)\right)^{-1}, z_{1}=\left\langle K \psi\left(j \omega_{e}\right)\right)^{-1} \underline{z}
\end{aligned}
$$

If $n=m$ results for the inverse return difference with the loop broken at the output are

$$
\begin{aligned}
& M=I+\left(\psi\left(j \omega_{e}\right) K\right)^{-1}=U E Z^{H} \\
& \frac{\partial \underline{\sigma}}{\partial t_{i j}}=-R e\left[U_{2}^{H} \frac{\partial K}{\partial t_{i j}} z_{2}\right] \\
& \frac{\partial \underline{\sigma}}{\partial \lambda_{i}}=-\operatorname{Re}\left[U_{2}^{H} \frac{\partial K}{\partial \lambda_{i}} z_{2}\right] \\
& U_{2}^{H}=\underline{u}^{H} K^{-1}, z_{2}=\left(\psi\left(j \omega_{e}\right) K\right)^{-1} \underline{z} \\
& \text { Appendix B }
\end{aligned}
$$

When some parameters $\lambda_{i}$ and $t_{i j}$ are complex numbers, expressions for the gradients are given below. The formula $(3-2)$ requires the parameter $p$ to be real $[9,12]$, 
this is accomplished by using the real and imaginary parts of $\lambda_{i}$ and $t_{i j}$ separately. The associated eigenspace is [1] (subscripts $R$ and $I$ denotes real and imaginary part of)

$$
\begin{aligned}
& v=\left[\begin{array}{llllll}
v_{1} & \cdots & v_{i R} & v_{i I} & \cdots & v_{n}
\end{array}\right] \\
& T=\left[\begin{array}{llllll}
t_{1} & \ldots & t_{i R} & t_{i I} & \cdots & t_{n}
\end{array}\right] \\
& v_{i R}=\Phi_{i R}\left(\lambda_{i}\right) B t_{i R}-\Phi_{i I}\left(\lambda_{i}\right) B t_{i I} \\
& v_{i I}=\Phi_{i I}\left(\lambda_{i}\right) B t_{i R}+\Phi_{i R}\left(\lambda_{i}\right) B t_{i I} \\
& \Phi_{i R}\left(\lambda_{i}\right)=\Phi_{i R}=\operatorname{Re} \Phi_{\left(\lambda_{i}\right)}, \Phi_{i I}\left(\lambda_{i}\right)=\Phi_{i I}=\operatorname{Im}\left(\lambda_{i}\right)
\end{aligned}
$$

Then

$$
\begin{aligned}
& M=I+K \psi\left(j \omega_{e}\right)=U \Sigma z^{H} \\
& \frac{\partial \underline{\sigma}}{\partial t_{i j R}}=\operatorname{Re}\left[\underline{\underline{u}} \frac{\partial K}{\partial t_{i j R}} \psi\left(j \omega_{e}\right) \underline{z}\right] \\
& \frac{\partial \underline{\sigma}}{\partial t_{i j I}}=\operatorname{Re}\left[\underline{u}^{H} \frac{\partial K}{\partial t_{i j I}} \psi\left(j \omega_{e}\right) \underline{z}\right]
\end{aligned}
$$

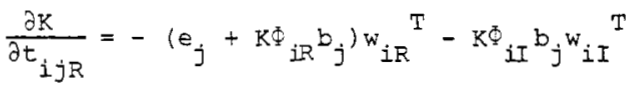

$$
\begin{aligned}
& \frac{\partial K}{\partial t_{i j I}}=K \Phi_{i I} b_{j} w_{i R}^{T}-\left(e_{j}+K \Phi_{i I} b_{j}\right) w_{i I}{ }^{T}
\end{aligned}
$$
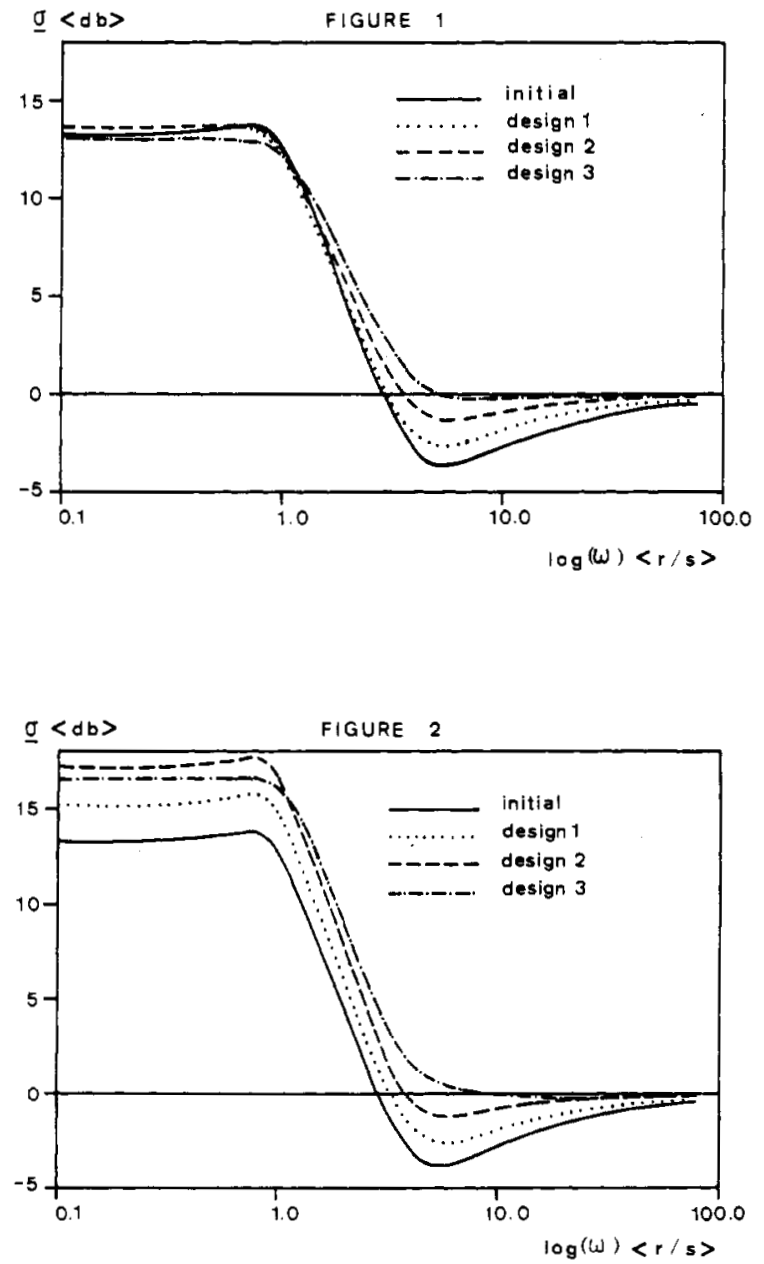

$$
\begin{aligned}
& \frac{\partial \underline{\underline{\sigma}}}{\partial \lambda_{i R}}=\operatorname{Re}\left[\underline{\underline{L}}^{\mathrm{H}} \frac{\partial \mathrm{K}}{\partial \lambda_{i R}} \psi\left(j \omega_{\mathrm{e}}\right) \underline{z}\right] \\
& \frac{\partial \underline{\underline{\sigma}}}{\partial \lambda_{i I}}=\operatorname{Re}\left[\underline{\underline{U}}^{\mathrm{H}} \frac{\partial \mathrm{K}}{\partial \lambda_{i I}} \psi\left(j \omega_{\mathrm{e}}\right) \underline{z}\right] \\
& \frac{\partial \mathrm{K}}{\partial \lambda_{i R}}=-\mathrm{K}\left[\frac{\partial \mathrm{v}_{i R}}{\partial \lambda_{i R}} w_{i R} \mathrm{~T}+\frac{\partial \mathrm{v}_{i I}}{\partial \lambda_{i R}} w_{i I} \mathrm{~T}\right] \\
& \frac{\partial K}{\partial \lambda_{i I}}=-K\left[\frac{\partial v_{i R}}{\partial \lambda_{i I}} w_{i R} T+\frac{\partial v_{i I}}{\partial \lambda_{i I}} w_{i I} \mathrm{~T}\right.
\end{aligned}
$$$$
\frac{\partial v_{i R}}{\partial \lambda_{i R}}=\left(\lambda_{i R} I-A\right)^{-1} \Phi_{i R} B t_{i I}-2 \Phi{ }_{i R} v_{i R}
$$$$
\frac{\partial v_{i I}}{\partial \lambda_{i R}}=\left(\lambda_{i R} I-A\right)^{-1} \Phi_{i R} B t_{i I}-2 \Phi_{i R} v_{i I}
$$$$
\frac{\partial v_{i R}}{\partial \lambda_{i I}}=-\Phi_{i I} B t_{i I} / \lambda_{i I}+2 \Phi_{i I} v_{i R}
$$$$
\frac{\partial v_{i I}}{\partial \lambda_{i I}}=-\Phi_{i I} B t_{i R} / \lambda_{i I}+2 \Phi_{i I} v_{i I}
$$

Similar expressions can be found for other robustness measures.

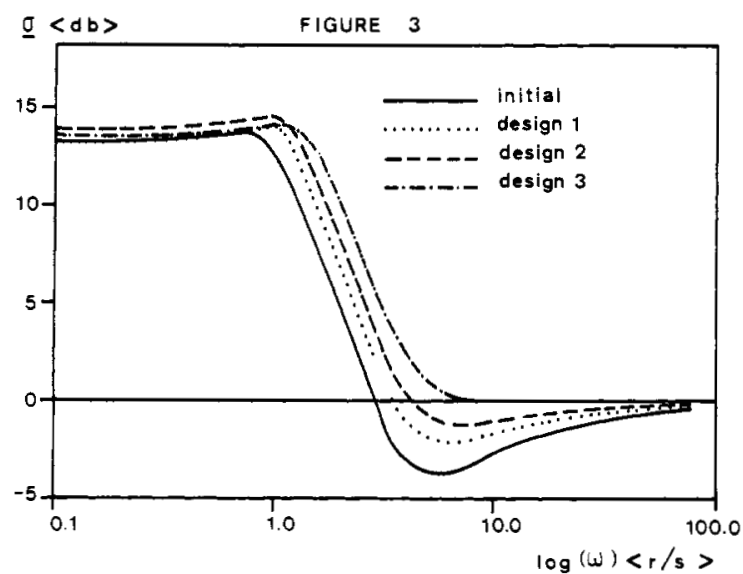

Figure 4

Gain margins

Phase margins

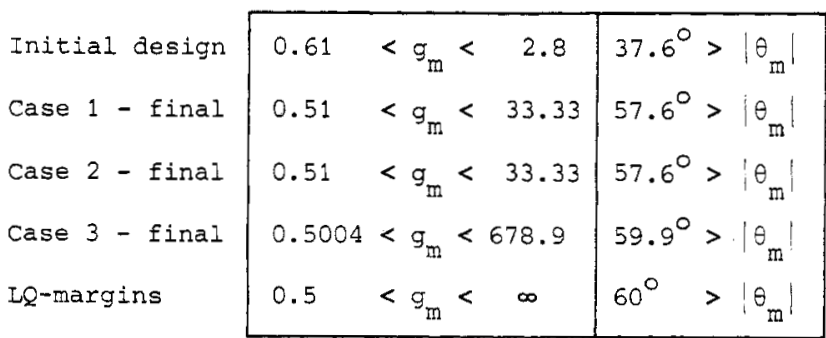

